\title{
Avaliação eletrocardiográfica em cães submetidos à pneumonectomia direita ${ }^{1}$
}

\author{
Eduardo T. Irino ${ }^{2}$, Angelo J. Stopiglia ${ }^{2^{*}}$, Maria H.M.A. Larsson ${ }^{3}$, Denise T. \\ Fantoni $^{4}$, Vera D. Aiello ${ }^{5}$, Márcia A.P. Kavhegian ${ }^{4}$, Edson A. Simões ${ }^{2}$, André \\ L.S. Santos ${ }^{2}$ e Huber A.N. Gama Filho ${ }^{2}$
}

\begin{abstract}
Irino E.T., Stopiglia A.J., Larsson M.H.M.A., Fantoni D.T., Aiello V.D., Kavhegian M.A.P., Simões E.A., Santos A.L.S. \& Gama Filho H.A.N. 2009. [Electrocardiographic evaluation in dogs submitted to right pneumonectomy.] Avaliação eletrocardiográfica em cães submetidos à pneumonectomia direita. Pesquisa Veterinária Brasileira 29(8):599604. Laboratório de Cirurgia Cardiotorácica, Departamento de Cirurgia, Faculdade de Medicina Veterinária e Zootecnia, Universidade de São Paulo, Av. Prof. Dr. Orlando Marques de Paiva 87, São Paulo, SP 05508-270, Brazil. E-mail: stopa@usp.br

The purpose for using lobectomy or pneumonectomy in dogs and cats, is to cure or palliate of bronchopulmonary diseases whenever conservative clinical treatment proves ineffective. Considering the marked changes caused by pneumonectomy, new experimental studies have to be done to assess the advantages of this surgical intervention allow its performance without any risk. The aim of the current study was to evaluate the electrocardiographic alterations in ten adult mongrel dogs of both sexes with between 10 and $30 \mathrm{Kg}$ that were submitted to right pneumonectomy. The clinical parameters of the dogs were evaluated in a daily basis and the alterations in every electrocardiogram derivation were recorded. All dogs presented a good post-operative outcome. In most cases there were no electrocardiographic alterations; when these alterations were observed they were of no clinical significance and included premature ventricular complexes in one dog, most likely resulting from a cardiorespiratory arrest that was reverted to successfully, and a decreased width in the QRS complex amplitude on the first 0-14 days post surgery which returned to normal after sixty days post surgery.
\end{abstract}

INDEX TERMS: Pneumonectomy, cardiology, electrocardiogram, dogs.

RESUMO.- O propósito das ressecções pulmonares em cães e gatos, quer sejam por lobectomia ou pneumonectomia, é a cura ou paliação de processos broncopulmonares sempre que os meios conservadores de tratamento clínico sejam considerados ineficientes. Tendo em vista as significativas alterações resultantes da pneumonectomia, novos estudos experimentais devem ser feitos para

\footnotetext{
${ }^{1}$ Recebido em 4 de julho de 2008.

Aceito para publicação em 9 de fevereiro de 2009.

${ }^{2}$ Laboratório de Cirurgia Cardiotorácica, Departamento de Cirurgia, Faculdade de Medicina Veterinária e Zootecnia (FMVZ), Universidade de São Paulo (USP), Av. Prof. Orlando Marques de Paiva 87, São Paulo, SP 05508-270, Brasil. *Autor para correspondência: stopa@usp.br

${ }^{3}$ Departamento de Clínica Médica, FMVZ-USP, São Paulo, SP.

${ }^{4}$ Departamento de Cirurgia, FMVZ-USP, São Paulo, SP.

5 Departamento de Anátomo-Patologia, Instituto do Coração, Faculdade de Medicina, USP, Av. Dr. Enéas de Carvalho Aguiar 44, São Paulo, SP 05403-000.
}

avaliar as vantagens dessa intervenção cirúrgica e determinar a maneira como aplicá-la com segurança. O presente estudo tem como objetivo avaliar as alterações eletrocardiográficas em dez cães adultos de ambos os sexos, sem raça definida, com $10-30 \mathrm{~kg}$, submetidos à pneumonectomia direita. Foram avaliados diariamente os parâmetros clínicos de cada cão e as alterações em todas as derivações do eletrocardiograma. Todos os cães apresentaram um bom desenlace pós-operatório. Apenas um cão apresentou alteração de relevância clínica, um caso de complexos ventriculares prematuros, possivelmente decorrente da parada cardiorrespiratória, que foi revertido com sucesso. Houve diminuição da amplitude dos complexos QRS nos primeiros 14 dias, retornado ao normal após 60 dias de pós-operatório.

TERMOS DE INDEXAÇÃO: Pneumonectomia, cardiologia, eletrocardiograma, cães. 


\section{INTRODUÇÃO}

Ressecções pulmonares em cães e gatos são realizadas visando cura ou paliação de processos broncopulmonares congênitos, neoplásicos, infecciosos ou traumáticos, sempre que os meios conservadores de tratamento clínico sejam considerados insuficientes (Walshaw 1994). Após pneumonectomia, todo o volume sangüíneo é enviado para um único pulmão, provocando aumento do fluxo nos vasos pulmonares e ocasionando a abertura ou distensão dos capilares, aumentando a capacidade de distribuição dos eritrócitos de forma mais homogênea, e cujo princípio também se aplica à ventilação (Takeda et al. 1999).

Pesquisas recentes demonstraram que a pneumonectomia esquerda e direita, do ponto de vista clínico-cirúrgico, é uma intervenção perfeitamente exeqüível (Irino et al. 2004, Simões et al. 2005). Estudos recentes sobre pneumonectomia, principalmente esquerda, em cães, determinaram as principais alterações histopatológicas localizadas no pulmão direito e as alterações eletrocardiográficas (Irino et al. 2004) ou compararam anatomopatologicamente sutura brônquica mecânica e manual (Simões et al. 2005). Altas taxas de mortalidade pós-operatória (Guggino et al. 2004, Jungraithmayr et al. 2004, Izquierdo Villarroya et al. 2005) e complicações trans e pós-operatórias (Krowka, et al. 1987, Eren et al. 2003, Foroulis et al. 2004, Liptak et al. 2004, Perigaud et al. 2004, Izquierdo Villarroya et al. 2005, Roselli et al. 2005) são relatadas em associação à pneumonectomia direita em humanos.

Alterações eletrocardiográficas resultantes da pneumonectomia têm sido descritas em humanos (Krowka et al. 1987, Frye \& Sahn 2000, Izquierdo Villarroya et al. 2005) e cães (Amar et al. 2002). Essas alterações incluem taquiarritmias como fibrilação atrial, flúter atrial, taquicardia supraventricular (Krowka et al. 1987, Frye \& Sahn 2000), aumento da freqüência cardíaca, desvio de eixo elétrico, aumento de amplitude da onda $\mathrm{P}$ e taquicardia ventricular (Frye \& Sahn 2000). Um dos relatos (Frye \& Sahn 2000) informa que $15 \%$ dos pacientes que apresentaram taquiarritmias morreram dentro dos 30 dias de pós-operatório. Arritmias ventriculares são consideradas raras no pós-operatório (Izquierdo Villarroya et al. 2005) de humanos e também não foram observadas na avaliação eletrocardiográfica de cães submetidos a pneumonectomia esquerda (Irino et al. 2004). No entanto, num estudo com 15 cães submetidos à pneumonectomia que visava associar idade avançada com incidência de arritmias supraventriculares, foi concluído que tal alteração ocorre devido principalmente à fibrose e inflamação atrial em cães com mais de oito anos de idade (Amar et al. 2002). Em humanos foi relatado que após pneumonectomia direita ocorre alteração mediastinal e elevação do diafragma, o que resulta em alteração na posição do coração de $31,4^{\circ}$ para a direita (Nonaka et al. 1999). O presente estudo teve como objetivo avaliar as alterações eletrocardiográficas em cães submetidos à pneumonectomia direita.

\section{MATERIAL E MÉTODOS}

O experimento foi conduzido de acordo com as normas da Comissão de Bioética da Faculdade de Medicina Veterinária e Zootecnia da Universidade de São Paulo (Protocolo n ${ }^{\circ} 476$ / 2004). Foram utilizados 10 cães, sem raça definida, de ambos os sexos, com 10-30 kg, provenientes de canis do Centro de Controle de Zoonoses de municípios localizados na Grande São Paulo (anterior à Lei Estadual no 11977).

Os cães incluídos no estudo foram avaliados por 60 dias; registros eletrocardiográficos foram obtidos nos seguintes momentos: 24 horas antes do ato operatório, 24 horas de pósoperatório e com sete, 14, 21, 36 e 60 dias de pós-operatório. Foram realizadas todas as derivações do plano frontal (DI, DII, DIII, aVR, aVL, aVF) e as pré-cordiais $\mathrm{CV}_{5} \mathrm{RL}, \mathrm{CV}_{6} \mathrm{LL}, \mathrm{CV}_{6} \mathrm{LU}$ e $\mathrm{V}_{10}$, obtidas uma de cada vez. Todas as mensurações foram realizadas na derivação frontal DII, ao qual, avaliou-se frequência cardíaca, eixo elétrico médio do QRS, amplitude e duração de onda $P$, complexo QRS e onda $T$, infradesnivelamento ou supradesnivelamento de segmento ST e medidas dos intervalos $\mathrm{P}-\mathrm{R}, \mathrm{Q}-\mathrm{T}$ e $\mathrm{S}-\mathrm{T}$. Os registros eletrocardiográficos foram interpretados por um único profissional integrante. Além dos momentos de avaliação especificados anteriormente, um único cão (Cão 7) também foi avaliado decorridas 12 horas do pós-operatório devido à parada cardiorrespiratória ocorrida após seis horas do ato operatório. Os dados foram submetidos à análise de variância para medidas repetidas seguida do teste de Dunn e comparação dos diferentes tempos de observação de um mesmo grupo. O grau de significância estabelecido para os dois testes estatísticos foi de $5 \%(p<0,05)$. Os parâmetros clínicos (freqüência cardíaca, freqüência respiratória, temperatura, coloração de mucosas, hidratação e dor) foram avaliados diariamente.

A medicação pré-anestésica constituiu de acepromazina $0,2 \%$ na dose $0,05 \mathrm{mg} / \mathrm{kg}$ associada ao cloridrato de meperidina na dose $2 \mathrm{mg} / \mathrm{kg}, 10$ minutos antes da indução. A indução anestésica foi realizada com propofol na dose de $5 \mathrm{mg} / \mathrm{kg}$ por via intravenosa. Quando atingido o plano anestésico adequado, o cão foi intubado com sonda de intubação seletiva. Realizouse manutenção anestésica com agente inalatório isofluorano para mantê-lo no $3^{\circ}$ plano de anestesia do $3^{\circ}$ estágio de Guedel. O bloqueio neuromuscular foi obtido com rocurônio na dose de $0,6 \mathrm{mg} / \mathrm{kg}$ por via endovenosa. A reversão do bloqueio ocorreu após toracorrafia com a administração de neostigmine na dose de $0,08 \mathrm{mg} / \mathrm{kg}$ por via intravenosa associado ao sulfato de atropina na dose de $0,04 \mathrm{mg} / \mathrm{kg}$.

O acesso cirúrgico do pulmão foi feito através de toracotomia lateral direita no quinto espaço intercostal. Os lobos pulmonares foram afastados dorsalmente permitindo a identificação das estruturas do hilo pulmonar. Inicialmente, foram identificadas as primeiras veias pulmonares direitas, responsáveis pela drenagem sanguínea dos lobos cranial e médio que receberam ligadura dupla com fio de náilon 2-0. Em seguida, identificou-se a veia pulmonar, responsável pela irrigação dos lobos caudal e acessório, localizados caudalmente ao brônquio, que recebeu a mesma ligadura dupla. Com a secção de todas as veias foi possível a visualização da artéria pulmonar direita, que foi ligada duplamente com fio náilon 2-0, pinçada com pinça hemostática não-traumática e seccionada. O coto resultante foi fechado em seguida com sutura do tipo colchoeiro duplo com fio de polipropileno 5-0. O brônquio principal direito foi pinçado e seccionado. Para o seu fechamento utilizou-se sutura manual com pontos simples "em oito" com fio de polipropileno 4-0. 
Após sutura, realizou-se minuciosa inspeção de campo operatório para verificar a eficácia na hemostasia e aerostasia. Para tanto foi introduzido solução fisiológica a $0,9 \%$ previamente aquecida. Não havendo borbulhamento, a solução salina foi aspirada. Um dreno tubular sob selo d'água foi colocado no espaço pleural, tendo sido mantido até que o cão apresentasse padrão respiratório estável.

Os cães receberam os seguintes cuidados pós-operatórios: analgesia, curativos da ferida cirúrgica e anotações dos parâmetros clínicos. Foram mantidos com colar elizabetano até a retirada dos pontos, 10 dias após o ato operatório. A analgesia foi mantida com a utilização de cloridrato de tramadol na dose de $1 \mathrm{mg} / \mathrm{kg}$ associado à dipirona $(25 \mathrm{mg} / \mathrm{kg})$ por sete dias.

\section{RESULTADOS}

Exceto pelo Cão 7, que apresentou hemotórax e evoluiu para parada cardiorrespiratória, ocorrida nas primeiras seis horas de pós-operatório, o período pós-operatório, desde imediato até 60 dias após intervenção cirúrgica foi sem intercorrências e com boa evolução clínica em todos os cães. A recuperação anestésica do Cão 7 foi demorada, quando comparado aos outros cães; após 6 horas, apresentou parada cardiorrespiratória, necessitando das manobras de ressuscitação cardiopulmonar e cerebral e transfusão de sangue. Apesar do êxito nesses procedimentos de ressuscitação, nova intervenção cirúrgica foi necessária, submetendo-o a toracotomia exploratória, por meio da qual se confirmou o hemotórax devido a sangramento difuso em cavidade torácica, oriundo principalmente do mediastino e da musculatura intercostal. Tal intercorrência possivelmente deveu-se à intensa trombocitopenia, cujo valor pré-operatório de 253.000 plaquetas $/ \mathrm{mm}^{3}$ de sangue caiu para 56.000 naquele momento. O eletrocardiograma de 12 horas pós-cirurgia constatou complexos ventriculares prematuros (Fig.1), que já não mais foram observados a partir do eletrocardiograma realizado 24 póscirurgia. O Cão 7 apresentou evolução clínica satisfatória. Decorridas 24 horas de pós-operatório, a melhora clínica estava evidente, com o cão apresentando normorexia, normodipsia, normoquesia, urina de coloração e volume normais e ausência de seqüelas neurológicas. Tal meIhora foi mantida até 60 dias de pós-operatório.

Noventa por cento dos cães mostraram diminuição da amplitude do complexo QRS, nas primeiras 24 horas de

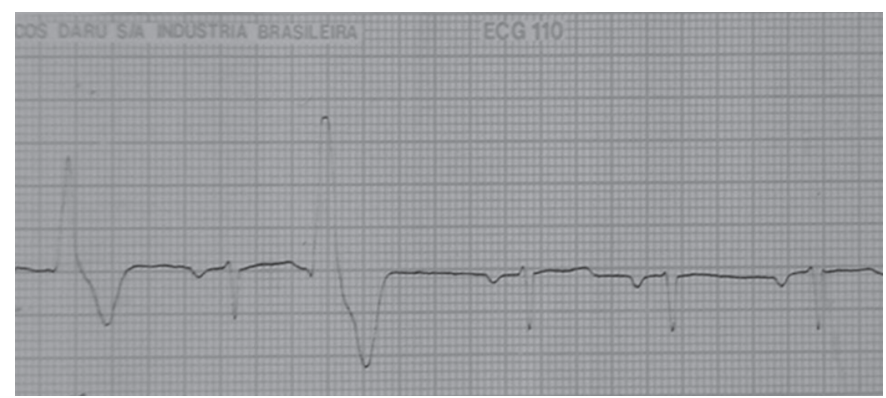

Fig.1. Registro eletrocardiográfico em derivação aVR (Cão 7). Presença de complexos ventriculares prematuros no momento de 12 horas de pós-operatório; v: 50mm/s.

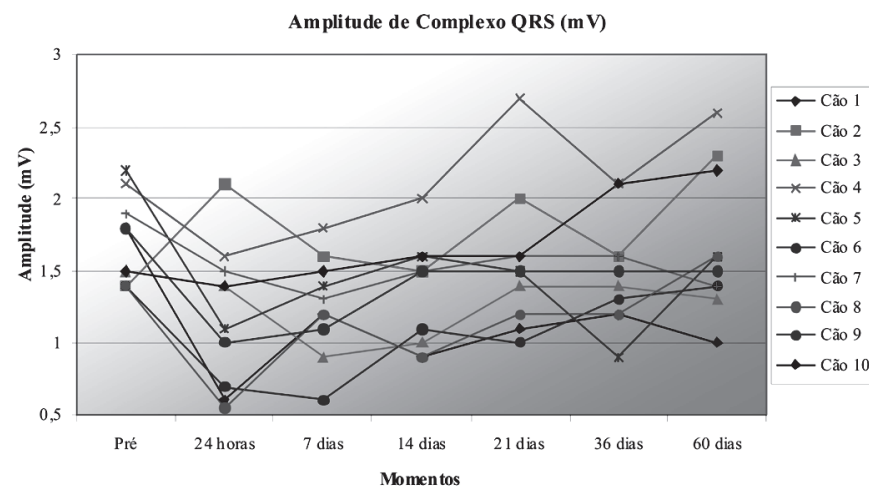

Fig.2. Valores da amplitude dos complexos QRS (mV) nos diferentes momentos dos cães submetidos à pneumonectomia direita.

Quadro 1. Valores da amplitude dos complexos QRS (mV) nos diferentes momentos dos 10 cães submetidos à pneumonectomia direita

Momentos Cão 1 Cão 2 Cão 3 Cão 4 Cão 5 Cão 6 Cão 7 Cão 8 Cão 9 Cão 10

$\begin{array}{ccccccccccc}\text { Préa }^{a} & 1,8 & 1,4 & 1,5 & 2,1 & 2,2 & 1,4 & 1,9 & 1,4 & 1,8 & 1,5 \\ 24 \text { horas }^{b} & 0,6 & 2,1 & 1,4 & 1,6 & 1,1 & 0,7 & 1,5 & 0,5 & 1,0 & 1,4 \\ 7 \text { dias }^{b} & 1,2 & 1,6 & 0,9 & 1,8 & 1,4 & 0,6 & 1,3 & 1,2 & 1,1 & 1,5 \\ 14 \text { dias }^{b} & 0,9 & 1,5 & 1,0 & 2,0 & 1,6 & 1,1 & 1,5 & 0,9 & 1,5 & 1,6 \\ 21 \text { dias }^{b} & 1,1 & 2,0 & 1,4 & 2,7 & 1,5 & 1,0 & 1,6 & 1,2 & 1,5 & 1,6 \\ 36 \text { dias }^{b} & 1,2 & 1,6 & 1,4 & 2,1 & 0,9 & 1,3 & 1,6 & 1,2 & 1,5 & 2,1 \\ 60 \text { dias }^{b} & 1,0 & 2,3 & 1,3 & 2,6 & 1,6 & 1,4 & 1,4 & 1,6 & 1,5 & 2,2\end{array}$

a Período pré-operatório; ${ }^{b}$ períodos pós-operatórios.

pós-operatório (Fig.2, Quadro 1). Vale ressaltar que tal alteração é bem visualizada nos Cães 1, 6 e 8; nesses, os valores diminuíram de $1,8 \mathrm{mV}, 1,4 \mathrm{mV}$ e $1,4 \mathrm{mV}$ para $0,6 \mathrm{mV}, 0,7 \mathrm{mV}$ e $0,5 \mathrm{mV}$, respectivamente (Fig.3). Nota-se ainda no Cão 6, que a diminuição da amplitude prossegue até 7 dias de pós-operatório, mantendo-se em 0,6mV.

Por meio de análise de variância com medidas repetidas observou-se que há alteração significativa ao longo das avaliações $(p=0,011)$ (Quadro 2). O momento préoperatório foi significantemente maior que os momentos 24 horas $(p=0,020), 7$ dias $(p=0,003)$ e 14 dias $(p=0,008)$. Porém, o momento pré-operatório não difere de maneira significativa dos momentos 21 dias $(p=0,361), 36$ dias $(p=0,217)$ e 60 dias $(p=0,957)$.
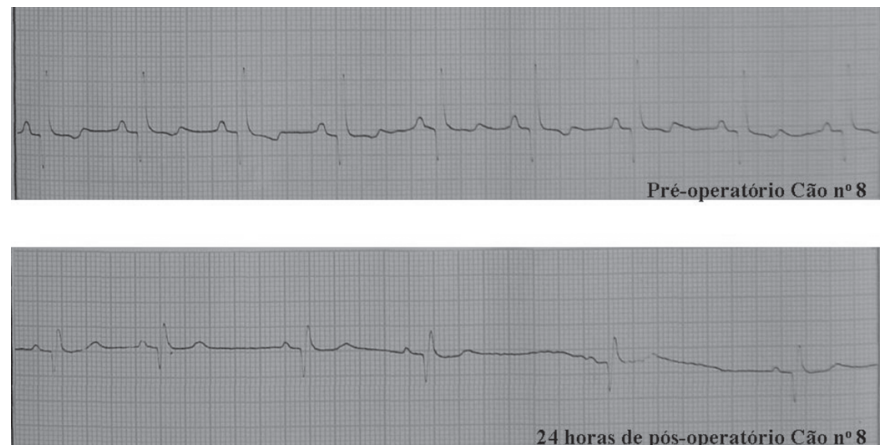

Fig.3. Registros eletrocardiográficos em derivação DII (Cão 8). Diminuição da amplitude de complexos QRS no momento de 24 horas de pós-operatório; v: 50mm/s. 
Quadro 2. Comparação da amplitude dos complexos QRS $(\mathrm{mV})$ nos diferentes momentos ${ }^{\mathrm{a}}$ dos 10 cães submetidos à pneumonectomia direita

\begin{tabular}{cccccc}
\hline Momentos & Média & DP $^{\mathrm{b}}$ & Mediana & Mínimo & Máximo \\
\hline Período pré-operatório & 1,70 & 0,30 & 1,65 & 1,40 & 2,20 \\
24 horas após a cirurgia & 1,19 & 0,50 & 1,25 & 0,50 & 2,10 \\
7 dias após a cirurgia & 1,26 & 0,35 & 1,25 & 0,60 & 1,80 \\
14 dias após a cirurgia & 1,36 & 0,37 & 1,50 & 0,90 & 2,00 \\
21 dias após a cirurgia & 1,56 & 0,49 & 1,50 & 1,00 & 2,70 \\
36 dias após a cirurgia & 1,49 & 0,38 & 1,45 & 0,90 & 2,10 \\
60 dias após a cirurgia & 1,69 & 0,51 & 1,55 & 1,00 & 2,60
\end{tabular}

a Por meio de análise de variância com medidas repetidas observou-se que houve alteração significativa $(p=0,011)$ ao longo das avaliações; b desvio padrão.

Cinqüenta por cento dos cães estudados apresentaram diminuição da freqüência cardíaca nas primeiras 24 horas de pós-operatório (Fig.4, Quadro 3). Tal diminuição é facilmente visualizada nos Cães 3, 5, 8 e 9, cujas freqüências caem $20 \%, 42 \%, 28 \%$ e $33 \%$, respectivamente. Entretanto, por meio de análise de variância com medidas repetidas, observou-se ausência de alterações significativas ao longo dos momentos avaliados $(p=0,077)$ conforme é mostrado no Quadro 4.

Observou-se ainda alteração do eixo elétrico em $60 \%$ dos cães. Porém, apenas os Cães 1 e 9 apresentaram uma alteração mais sensível. O Cão 1 estava no eixo $30^{\circ}$ a $60^{\circ}$ e passou para $-30^{\circ}$ e o Cão 2 inverteu de $90^{\circ}$ a $120^{\circ}$ para $-90^{\circ}$ a $-120^{\circ}$. Tal alteração permaneceu até os 60

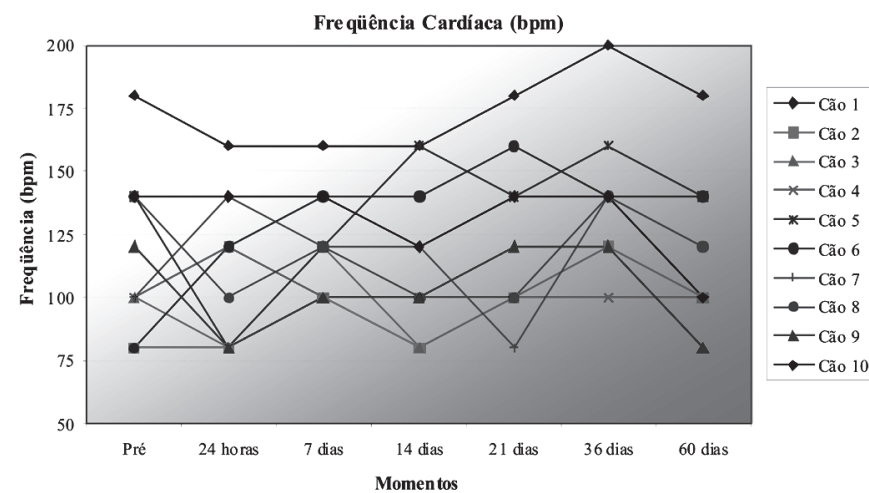

Fig.4. Valores da freqüência cardíaca (batimentos por minuto) nos diferentes momentos dos cães submetidos à pneumonectomia direita.

Quadro 3. Valores da freqüência cardíaca (batimentos por minuto) nos diferentes momentos dos cães submetidos à pneumonectomia direita

Momentos Cão 1 Cão 2 Cão 3 Cão 4 Cão 5 Cão 6 Cão 7 Cão 8 Cão 9 Cão 10

\begin{tabular}{ccccccccccc}
\hline Préa $^{\mathrm{a}}$ & 180 & 80 & 100 & 100 & 140 & 80 & 100 & 140 & 120 & 140 \\
24 horas $^{\mathrm{b}}$ & 160 & 80 & 80 & 120 & 80 & 120 & 140 & 100 & 80 & 140 \\
7 dias $^{\mathrm{b}}$ & 160 & 100 & 120 & 100 & 120 & 140 & 120 & 120 & 100 & 140 \\
14 dias $^{\mathrm{b}}$ & 160 & 80 & 80 & 100 & 160 & 140 & 120 & 100 & 100 & 120 \\
21 dias $^{\mathrm{b}}$ & 180 & 100 & 100 & 100 & 140 & 160 & 80 & 100 & 120 & 140 \\
36 dias $^{\mathrm{b}}$ & 200 & 120 & 120 & 100 & 160 & 140 & 140 & 140 & 120 & 140 \\
60 dias $^{\mathrm{b}}$ & 180 & 100 & 100 & 100 & 140 & 140 & 120 & 120 & 80 & 100
\end{tabular}

a Período pré-operatório; ${ }^{b}$ períodos pós-operatórios.
Quadro 4. Comparação da freqüência cardíaca (bpm) nos diferentes momentos ${ }^{a}$ dos cães submetidos à pneumonectomia direita

\begin{tabular}{cccccc}
\hline Momentos & Média & DPb & Mediana & Mínimo & Máximo \\
\hline Período pré-operatório & 118 & 31,90 & 110 & 80 & 180 \\
24 horas após a cirurgia & 110 & 30,18 & 110 & 80 & 160 \\
7 dias após a cirurgia & 122 & 19,89 & 120 & 100 & 160 \\
14 dias após a cirurgia & 116 & 29,51 & 110 & 80 & 160 \\
21 dias após a cirurgia & 122 & 31,90 & 110 & 80 & 180 \\
36 dias após a cirurgia & 138 & 27,41 & 140 & 100 & 200 \\
60 dias após a cirurgia & 118 & 28,98 & 110 & 80 & 180
\end{tabular}

a Por meio de análise de variância com medidas repetidas observou-se que houve alteração significativa $(p=0,077)$ ao longo das avaliações; b desvio padrão.

dias de pós-operatório. Outra alteração eletrocardiográfica observada foi o bloqueio sinoatrial, que ocorreu no Cão 9, no sétimo dia pós-operatório, com normalização do ritmo normalizou nos períodos seguintes. Marcapasso migratório ocorreu em $80 \%$ dos animais operados. Não foram observadas alterações nas medidas de onda P, complexo QRS, onda T, segmento PR, QT e ST.

\section{DISCUSSÃO}

Dados de medicina humana indicam altos índices de morbidade (40\%) e mortalidade (12\%) associados à pneumonectomia (Izquierdo Villarroya et al. 2005). Neste trabalho, todos os cães manifestaram satisfatória evolução clínica pós-operatória, inclusive o Cão 7, que apresentou intercorrências pós-operatórias. No entanto, deve-se ressaltar que neste estudo foram utilizados cães hígidos, que foram avaliados num período pós-operatório curto (60 dias) se comparado aos períodos de avaliação em estudos em medicina humana que já afirmavam que o risco, em humanos, é alto porque grande parte das complicações trans e pós-operatórias ocorrem asssociadas à doenças cardíacas e respiratórias obstrutivas (Liptak et al. 2004). Em medicina veterinária essas complicações são mais freqüentes em cães de mais de oito anos e estão associadas à fibrose, miocardite e epicardite atrial (Amar et al. 2002). Neste experimento os cães tinham menos de quatro anos de idade e a análise histológica estava fora dos objetivos do trabalho.

Em uma pesquisa com seis cães submetidos à pneumonectomia direita, um cão morreu imediatamente após a intervenção cirúrgica por edema pulmonar agudo e outros dois morreram no decorrer do experimento, no entanto, sem terem descrito a causa e o momento da morte destes (Takeda et al. 1999). Em estudo realizado em pacientes humanos acometidos de grave traumatismo torácico (Wagner et al. 1996), o índice de mortalidade foi próximo a $100 \%$. Porém, em pacientes submetidos à pneumonectomia de emergência, há índice de sobrevivência de $56 \%$ quando se utiliza sutura mecânica com grampeador e $33 \%$ quando se utiliza sutura manual. A casuística desses traumas torácicos em medicina veterinária de pequenos animais sugere que um fator que pode 
contribuir para o aumento dos índices de mortalidade é a baixa massa corpórea, que deixa os cães mais vulneráveis às hemorragias agudas e posterior choque hipovolêmico.

As complicações pós-operatórias causadoras de insuficiência respiratória mais freqüentemente citadas na literatura são pneumonia, edema pulmonar, tromboembolismo pulmonar, fístula broncopleural, piotórax, fístula esofagopleural, quilotórax, hemotórax e hipertensão pulmonar (Frye \& Sahn 2002, Izquierdo Villarroya et al. 2005). Neste estudo houve um caso de hemotórax seguido de parada cardiorrespiratória. O edema pulmonar é outra grave e freqüente complicação após pneumonectomias. Estudos apontam para incidência de 7,1\% para pneumonectomia direita e 2,3\% para esquerda, fato que ocorre com mais freqüência nas primeiras 24-72 horas de pósoperatório. Adicione-se ainda a alta mortalidade associada ao edema pulmonar, alcançando índices de $50 \%$, dependendo da gravidade do quadro (Izquierdo Villarroya et al. 2005). Em um estudo (Takeda et al. 1999), um dos cães morreu imediatamente após pneumonectomia direita devido a edema pulmonar agudo.

A intervenção cirúrgica empregada neste estudo tem sido referida na literatura como técnica utilizada para investigar as origens e a extensão da capacidade de adaptação pulmonar em pacientes acometidos de enfermidades pulmonares, pois simula as afecções caracterizadas por perda física ou funcional dos alvéolos (Takeda et al. 1999). Fato que concordamos, principalmente quanto à compensação respiratória, pois todos os cães apresentaram evolução clínica satisfatória.

A freqüência cardíaca de pacientes humanos submetidos à pneumonectomia apresentou significativo aumento após os 30 dias pós-operatórios, passou de 75+3,7 bpm para 100+5,5 bpm (Frye \& Sahn 2000). Os mesmos autores ainda acrescentaram ser, a taquicardia sinusal, achado consistente em pós-operatório de pneumonectomia, cujas causas mais freqüente são dor, hipóxia, anemia, febre, hipovolemia, hipervolemia, hipotensão, embolia pulmonar, infecção e distúrbios hidroeletrolíticos. Porém, tal fato não foi presenciado neste estudo, ao qual foi observado o inverso: redução da freqüência cardíaca nas primeiras 24 horas de pós-operatório em $50 \%$ dos cães. A ausência das complicações citadas acima pode ser colocada como um dos fatores que contribuíram para estes resultados serem diferentes do que é citado na literatura.

Das arritmias, as mais comuns são a fibrilação atrial, taquicardia supraventricular e flúter atrial. (Krowka et al. 1987, Von Knorring et al. 1992, Curtis et al. 1998, Frye \& Sahn 2000, Rena et al. 2001, Izquierdo Villarroya et al. 2005, Roselli et al. 2005). Alguns autores (Toro lbanez et al. 1999) relataram que $97,3 \%$ das arritmias encontradas são supraventriculares e que $55,3 \%$ dessas são por fibrilação atrial. Outros autores (Park et al. 2007) ainda acrescentam que a fibrilação atrial ocorre nas intervenções cirúrgicas pulmonares, tanto quando o acesso é por toracotomia convencional quanto por toracoscopia vídeo- assistida. Nesse estudo, poucas foram as alterações no ritmo cardíaco, podendo citar apenas um caso de bloqueio sinoatrial, manifestado nas primeiras 24 horas de pósoperatório, sem, no entanto, o cão apresentar qualquer manifestação de afecção cardiovascular.

A ocorrência de complexos ventriculares prematuros no Cão 7, que apresentou parada cardiorrespiratória seis horas após a intervenção cirúrgica, provavelmente está mais relacionada com a parada cardiorrespiratória ocorrida do que com o fato do cão ter sido submetido à pneumonectomia direita. Arritmias ventriculares são freqüentes em situações que exigem as manobras de ressuscitação cardiopulmonar e cerebral, sobretudo os complexos ventriculares prematuros (Borde \& Dhupa 2002)

Foi relatado anteriormente (Irino et al. 2004) que as principais alterações eletrocardiográficas ocorriam em período pós-operatório mais tardio (36 dias), ao contrário do observado por nós, cujos achados mais relevantes ocorreram nas primeiras 24 horas de pós-operatório. Um achado consistente deste estudo foi a diminuição da amplitude do complexo QRS nas primeiras 24 horas de pósoperatório em $90 \%$ dos cães. Não foram encontrados relatos na literatura relacionando essa alteração à pneumonectomia. No entanto, é descrito que diminuição da amplitude do complexo QRS pode ocorrer em casos de pneumotórax (Goodwin 2002), condição que ocorre ao final do procedimento de pneumonectomia, durante a toracorrafia. Esse episódio de pneumotórax é prontamente revertido, mas pode ser uma possível causa deste achado eletrocardiográfico.

A principal alteração eletrocardiográfica observada no pós-operatório da pneumonectomia esquerda foi o desvio de eixo elétrico em $83 \%$ dos cães testados (Irino et al. (2004). Neste estudo percebemos tal alteração eletrocardiográfica em $60 \%$ dos cães. Isso sugere que tanto a pneumonectomia esquerda quanto a direita induz uma reorganização espacial no tórax, em conseqüência da formação de um grande espaço morto, outrora ocupado pelo pulmão removido. Alteração do de eixo elétrico $\left(31,4^{\circ} \mathrm{em}\right.$ média) após lobectomia ou pneumonectomia devido à mudança mediastinal e elevação do diafragma resultando em mudança da posição do coração já foi relatada (Nonaka et al. 1999).

\section{CONCLUSÕES}

A avaliação dos resultados obtidos permite concluir que as alterações eletrocardiográficas ocorridas no período pós-operatório de até 60 dias da pneumonectomia direita não apresentaram relevância clínica, pois a grande maioria dos cães demonstrou evolução satisfatória. Entretanto, a parada cardiorrespiratória pode ocorrer devido a complicações decorrentes do procedimento, o que reforça a necessidade de monitoramento e cuidados intensivos no pós-operatório imediato.

Agradecimentos.- À Fundação de Amparo à Pesquisa do Estado de São Paulo (FAPESP) pelo apoio financeiro à realização deste estudo. 


\section{REFERÊNCIAS}

Amar D., Heerdt P.M., Korst R.J., Zhang H. \& Nguyen H. 2002. The effects of advanced age on the incidence of supraventricular arrhythmias after pneumonectomy in dogs. Anesthesia and Analgesia 94(5):1132-1136.

Borde D.J. \& Dhupa N.D. 2002. Parada e ressuscitação cardiopulmonar, p.377-393. In: Tilley L.P. \& Goodwin J.K. (Eds), Manual de Cardiologia para Cães e Gatos. Roca, São Paulo. 489p.

Curtis J.J., Parker B.M., McKenney C.A., Wagner-Mann C.C., Walls J.T., Demmy T.L. \& Schmaltz R.A. 1998. Incidence and predictors of supraventricular dysrhythmias after pulmonary resection. Annals Thoracic Surg. 66(5):1766-1771.

Eren S., Eren M.N. \& Balci A.E. 2003. Pneumonectomy in children for destroyed lung and the long-term consequences. J. Thoracic Cardiovasc. Surg. 126(2):574-581.

Foroulis C.N., Kotoulas C.S., Kakouros S., Evangelatos G., Chassapis C., Konstantinou M. \& Lioulias A.G. 2004. Study on the late effect of pneumonectomy on right heart pressures using Doppler echocardiography. Eur. J. Cardiothor. Surg. 26(3):508-514.

Goodwin J.K. 2002. Eletrocardiografia, p.39-65. In: Tilley L.P. \& Goodwin J.K. (Eds), Manual de Cardiologia para Cães e Gatos. Roca, São Paulo. 489p.

Frye D.M. \& Sahn A.S. 2000. Acute electrocardiographic changes after pneumonectomy. Southern Med. J. 93(10):977-981.

Guggino G., Doddoli C., Barlesi F., Acri P., Chetaille B., Thomas P., Giudicelli R. \& Fuentes P. 2004. Completion pneumonectomy in cancer patients: experience with 55 cases. Eur. J. Cardiothor. Surg. 25(3):449455.

Irino E.T., Stopiglia A.J., Larsson M.H.M.A., Guerra J.L., Simões E.A., Shinkai M.T., Fantoni D.T., Otsuki D.A., Freitas R.R., Saldiva P.H.N. \& Jatene F.B. 2004. Avaliação histopatológica do pulmão direito e avaliação eletrocardiográfica de cães submetidos à pneumonectomia esquerda. Braz. J. Vet. Res. Anim. Sci. 48(1):58-66.

Izquierdo Villarroya B., Lopez Alvarez S., Bonome Gonzales C. \& Cassinello Ogea C. 2005. Cardiovascular and respiratory complications after pneumonectomy. Revta Española Anestesiol. Reanimación 52(8):474-489.

Jungraithmayr W., Hasse J., Olschewski M. \& Stoelben E. 2004. Indications and results of completion pneumonectomy. Eur. J. Cardiothor. Surg. 26(1):189-196.

Krowka M.J., Pairolero P.C., Trastek V.F., Payne W.S. \& Bernatz P.E. 1987. Cardiac dysrhythmia following pneumonectomy: Clinical correlates and prognostic significance. Chest 91(4):490-495.
Liptak J.M., Monnet E., Dernell W.S., Rizzo S.A. \& Withrow S.J. 2004. Pneumonectomy: Four cases studies and a comparative review. J. Small Anim. Pract. 45(9):441-447.

Nonaka M., Kadokura M., Yamamoto S., Tanio N., Kataoka D., Kawada T. \& Takaba T. 1999. Lung resection affects the postoperative arrhythmia and electrocardiographic axis deviation. Kyobu Geka. Japn. J. Thoracic Surg. 52(9):739-741.

Park B.J., Zhang H., Rusch V.W. \& Amar D. 2007. Video-assisted thoracic surgery does not reduce the incidence of postoperative atrial fibrillation after pulmonary lobectomy. J. Thoracic Cardiovasc. Surg. 133(3):775779.

Perigaud C., Baron O., Roussel J.C., Al Habash O., Despins P., Michaud J.L. \& Duveau D. 2004. Pneumothorax after left pneumonectomy: Implantation of an intrapleural prosthesis. Annals Thoracic Surg. 78(2):707-709.

Rena O., Papalia E., Oliaro A., Casadio C., Ruffinim E., Filosso P., Sacerdote C. \& Maggi G. 2001. Supraventricular arrhythmias after resection surgery of the lung. Eur. J. Cardiothor. Surg. 20(4):688693.

Roselli E.E., Murthy S.C., Rice T.W., Houghtaling P.L., Pierce C.D., Karchmer D.P. \& Blackstone E.H. 2005. Atrial fibrillation complicating lung cancer resection. J. Thoracic Cardiovasc. Surg. 130(2):438-444.

Simões E.A., Jatene F.B., Stopiglia A.J., Fantoni D.T., Guerra J.L., Aiello V.D., Monteiro R., Irino E.T., Otsuki D.A., Binoki D.H., Freitas R.R. \& Unruh S.M. 2005. Estudo comparativo entre sutura mecânica e manual em brônquio após pneumonectomia esquerda em cães (Canis familiaris): uma avaliação anatomopatológica. Braz. J. Vet. Res. Anim. Sci. 42(5):357-366.

Takeda S.I., Hsia C.C.W., Wagner E., Ramanathan M., Estrera A.S. \& Weibel E.R. 1999. Compensatory alveolar growth normalizes gasexchange function in immature dogs after pneumonectomy. J. Appl. Physiol. 86(4):1301-1310.

Toro Ibanez D., Casielles Garcia J.L \& De Las Mulas Bejar M. 1999. Arrhythmias in the pulmonary exeresis postoperative period. Revta Española Anestesiol. Reanimación 46(1):9-13.

Von Knorring J., Lepantalo M., Lindgren L. \& Lindfors O. 1992. Cardiac arrhythmias and myocardial ischemia after thoracotomy for lung cancer. Annals Thoracic Surg. 53(4):642-647.

Wagner J.W., Obeid F.N., Karmy-Jones R.C., Casey G.D., Sorensen V.J. \& Horst H.M. 1996. Trauma pneumonectomy revisited: The role of simultaneously stapled pneumonectomy. J. Trauma 40(4):590-594.

Walshaw R. 1994. Stapling techniques in pulmonary surgery. Vet. Clin. North Am., Small Anim. Pract. 24(2):335-366. 